\title{
Inégalités sociales des décès liés à la COVID-19 au Canada, par caractéristiques individuelles et locales, de janvier à juillet/août 2020 : résultats de deux processus nationaux d'intégration de données
}

\author{
Alexandra Blair ${ }^{1 \star}$, Sai Yi Pan², Rajendra Subedi ${ }^{3}$, Fei-Ju Yang $^{3}$, Nicole Aitken ${ }^{3}$, Colin Steensma ${ }^{1}$
}

\section{Résumé}

Contexte : Malgré les premiers rapports sur les déterminants sociaux de l'infection par le coronavirus du syndrome respiratoire aigu sévère 2 (SRAS-CoV-2) et de la maladie à coronavirus 2019 (COVID-19), les analyses sur les inégalités attribuables à la COVID-19 au niveau national ont été limitées. L'objectif de cette étude est de décrire les inégalités en matière des décès attribuables à la COVID-19 au Canada en utilisant des données préliminaires obtenues dans le cadre de l'Initiative pancanadienne sur les inégalités en santé.

Méthodes : Deux intégrations des données provisoires de la Base canadienne de données sur les décès de la Statistique de l'état civil ont été utilisées. Les données sur les décès survenus entre le $1^{\text {er }}$ janvier et le 4 juillet 2020 parmi les résidents de logements privés ont été liées aux données, au niveau individuel, provenant du questionnaire abrégé du Recensement de 2016. Cette intégration a permis de ventiler les données de mortalité selon le sexe et la situation de faible revenu, le type de logement, le type de ménage et la taille du ménage. Les données sur

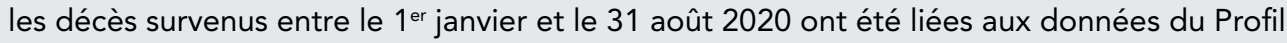
de Recensement au niveau local, du Recensement de 2016. Cette intégration de données a permis de ventiler des données de mortalité selon le sexe et les caractéristiques des quartiers, dont les ont été ventilées par sexe et par quintiles de composition ethnoculturelle (déterminés en fonction de la proportion de personnes ayant immigré récemment, appartenant à une minorité visible, étant nées à l'extérieur du Canada, ne connaissant ni l'anglais ni le français), par quintiles du revenu et par résidence dans une région métropolitaine. Les différences et les ratios des taux de mortalité liée à la COVID-19 normalisés selon l'âge (pour 100000 habitants) entre groupes ont été estimés.

Résultats : En juillet/août 2020, les résidents d'appartement, des grands centres urbains, des quartiers ayant la plus forte concentration de composition ethnoculturelle ou des quartiers ayant les plus faibles revenus ont connu 14 à 30 décès liés à la COVID-19 pour 100000 habitants de plus comparativement aux groupes de référence (résidents d'une maison individuelle non attenante, ceux qui se trouvent en dehors des centres urbains, ceux résidant dans des quartiers avec la concentration de composition ethnoculturelle la plus faible ou ceux détenant les revenus les plus élevés, respectivement). Les inégalités entre les sexes étaient également plus importantes dans ces quatre groupes (11 à 18 décès chez les hommes de plus que chez les femmes pour 100000 habitants) que dans les groupes de référence ( 2 à 4 décès chez les hommes de plus que chez les femmes pour 100000 habitants).

Conclusion : Ces résultats reflètent le fardeau global de mortalité plus élevé que subissent les populations défavorisées au niveau socioéconomique. Les domaines d'études futures sont abordés dans l'optique de guider une réponse à la pandémie axée sur l'équité en santé.
Cette oeuvre est mise à la disposition selon les termes de la licence internationale Creative Commons Attribution 4.0

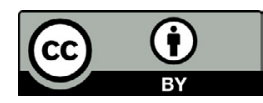

Affiliations

1 Division des déterminants sociaux de la santé, Agence de la santé publique du Canada, Montréal, QC

2 Division des déterminants sociaux de la santé, Agence de la santé publique du Canada, Ottawa, ON

${ }^{3}$ Statistiques Canada, Ottawa, ON

${ }^{\star}$ Correspondance : alexandra.blair@phac-aspc.gc.ca 
Citation proposée : Blair A, Pan SY, Subedi R, Yang F-J, Aitken N, Steensma C. Inégalités sociales des décès liés à la COVID-19 au Canada, par caractéristiques individuelles et locales, de janvier à juillet/août 2020 : résultats de deux processus nationaux d'intégration de données. Relevé des maladies transmissibles au Canada 2022;48(1):30-42. https://doi.org/10.14745/ccdr.v48i01a05f

Mots-clés : SRAS-CoV-2, COVID-19, mortalité, déterminants sociaux de la santé, équité en santé, Canada

\section{Introduction}

Les premiers rapports régionaux (1-3), provinciaux $(4,5)$ et nationaux $(6,7)$ au Canada ont indiqué que le fardeau de la maladie à coronavirus 2019 (COVID-19) n'a pas été réparti également chez la population canadienne. De plus, des analyses bivariées suggèrent que les populations racisées et à faible revenu ont connu des taux d'infection et de mortalité liées à la COVID-19 plus élevés que les groupes non-racisés ou aux revenus les plus élevés, dans plusieurs juridictions canadiennes. $(1,2,7)$ Ces études soulignent l'importance des conditions sociales et économiques, connues collectivement sous le nom de déterminants sociaux de la santé (8), dans la distribution des infections par le coronavirus du syndrome respiratoire aigu sévère 2 (SRAS-CoV-2) et de la morbidité et de la mortalité liées à la COVID-19 $(9,10)$.

Plusieurs hypothèses ont été proposées pour expliquer les inégalités en matière de mortalité liée à la COVID-19, chacune étant liée aux déterminants sociaux de la santé sous-jacents (9). Premièrement, elles peuvent être dues à des inégalités dans les infections par le SRAS-CoV-2 en raison d'inégalités sociales et économiques systémiques dans les conditions de vie ou de travail $(9,11,12)$ dans lesquelles les stratégies de prévention, telles que la distanciation physique ou l'amélioration de la ventilation, sont plus difficiles à appliquer ou n'ont pas été mises en œuvre (13-15). Deuxièmement, elles peuvent être attribuables à des inégalités sociales et économiques persistantes (11) au niveau de la prévalence de conditions et de comportements à risque, tels que le tabagisme, l'obésité ou le diabète, qui exposent les populations défavorisées sur le plan socioéconomique à un risque plus élevé de morbidité liée à la COVID-19 (16). Troisièmement, elles peuvent être attribuables à des inégalités socioéconomiques en matière d'accès, d'utilisation et de qualité des soins de santé $(9,11,17)$.

La déclaration à l'échelle nationale des cas de mortalité liée à la COVID-19 par groupes socioéconomiques au Canada reste limitée (18) et ce, malgré le besoin exprimé par les chercheurs et les collectivités (19-21) de contribuer à une préparation et une réponse à la pandémie axées sur l'équité. Pour combler cette lacune dans la déclaration des données nationales liées à la COVID-19, la présente analyse avait l'objectif de synthétiser les inégalités absolues et relatives selon des mesures individuelles et locales en matière de mortalité liée à la COVID-19 entre janvier et août 2020. Ce rapport s'insère dans l'effort continu de l'Initiative pancanadienne sur les inégalités en matière de santé (11).

\section{Méthodes}

\section{Sources de données}

Les données de ce rapport proviennent de deux intégrations de données réalisées par Statistique Canada (pour lesquelles l'équipe d'intégration des données était formée notamment des co-auteurs R. S., F. J. Y., N. A.). Le processus d'intégration des données de Statistique Canada fait référence à la combinaison de deux ou plusieurs ensembles de données. Les intégrations de données décrites ici ont été réalisées dans le contexte de la pandémie de COVID-19 pour informer plusieurs études, dont celle-ci. L'une des intégrations réalisées s'est faite entre la Base canadienne de données provisoire sur les décès de la Statistique de l'état civil et le Recensement de la population de 2016 du Canada, questionnaire abrégé (22). Ce couplage d'enregistrements au niveau individuel a été jumelé de façon déterministe au Dépôt d'enregistrements dérivés dans l'Environnement de couplage de données sociales de Statistique Canada (23). L'Environnement de couplage de données sociales est décrit comme un « environnement hautement sécurisé qui facilite la création de fichiers de données couplées sur la population à des fins d'analyse sociale. Il ne s'agit pas d'une grande base de données intégrées » (23). En tout, 96.4\% des enregistrements provisoires de décès signalés entre le $1^{\text {er }}$ janvier et le 4 juillet 2020 ont été jumelés de façon déterministe au Dépôt d'enregistrements dérivés de l'Environnement de couplage de données sociales. Le taux de couplage des répondants du questionnaire abrégé du Recensement avec le dépôt d'enregistrements dérivés était de 96,8 \%. Cette source de données couplée au recensement de la Base canadienne de données provisoire sur les décès de la Statistique de l'état civil comprend les décès liés à la COVID-19 survenus entre le $1^{\mathrm{er}}$ janvier et le 4 juillet 2020, pour les résidents de logements privés, ce qui représente $98 \%$ de la population canadienne ( $N=4430$ décès; 1990 femmes, 2440 hommes; ici, le nombre de décès est arrondi, conformément aux règles relatives à la divulgation de Statistique Canada) $(6,22,24)$. Les décès survenus dans des logements collectifs, y compris les soins de longue durée, ont été exclus.

L'autre intégration de données s'est faite entre la Base canadienne de données sur les décès de la Statistique de l'état civil et les mesures à l'échelle du quartier, grâce aux renseignements géographiques supplémentaires fournis sur le Fichier de conversion des codes postaux plus (FCCP+) de 2016 (25). Cette source de données liée Base canadienne de données provisoire sur les décès de la Statistique de l'état civil et FCCP+ comprend les décès liés à la COVID-19 survenus entre le 


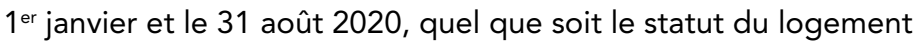
(total arrondi de 9265 décès liés à la COVID-19; 4990 femmes, 4275 hommes). Parmi les dossiers de décès liés à la COVID-19 signalés entre le $1^{\mathrm{er}}$ janvier et le 31 août 2020, 99,7 \% avaient des codes postaux trouvés dans le FCCP+.

Les données de la Base canadienne de données sur les décès de la Statistique de l'état civil sont provisoires et incomplètes pour plusieurs raisons. En effet, cet ensemble de données est sensible aux délais de déclaration des provinces et territoires et exclut les décès survenus au Yukon. Cependant, les taux de mortalité liée à la COVID-19 estimés à l'aide des données provisoires de l'état civil sont relativement similaires (à plus ou moins de $5 \%$ ) à ceux obtenus à l'aide des données de surveillance de la COVID-19 (22). De plus, les caractéristiques des individus enregistrées lors du Recensement de 2016 peuvent avoir changé au moment où les décès ont été enregistrés en 2020. Néanmoins, ces intégrations sont les meilleures sources disponibles de données nationales canadiennes concernant les caractéristiques socioéconomiques des décès liés à la COVID-19. Ces données peuvent fournir des éléments probants préliminaires sur les questions de santé publique émergentes afin $d$ 'orienter les recherches futures. Elles fournissent également des renseignements de base sur lesquels fonder le suivi futur.

\section{Mesures}

La mesure dépendante étudiée était la mortalité liée à la COVID-19, opérationnalisée en tant que taux de mortalité cumulatif normalisé selon l'âge pour 100000 habitants (ci-après dénommé le « taux de mortalité pour 100000 habitants »; des renseignements plus détaillés concernant la normalisation sont fournis ci-dessous). La Base canadienne de données sur les décès de la Statistique de l'état civil identifie les décès liés à la COVID-19 en se basant sur les certificats de décès sur lesquels la COVID-19 figure comme cause sous-jacente du décès. Les codes U071 et U072 de la CIM-10 ont été utilisés pour identifier, respectivement, les décès parmi les personnes ayant reçu un résultat positif au test de dépistage du SRAS-CoV-2 (quel que soit le test de laboratoire utilisé) et les personnes identifiées comme des cas "potentiels » ou " probables », ou qui étaient " en attente d'un résultat de test (positif)».

Sept mesures de stratification ont été utilisées pour étudier les déterminants sociaux de la santé connus, tels qu'identifiés dans le cadre des déterminants sociaux de la santé (8). À partir de l'intégration de Statistique provisoire de l'état civil et du questionnaire abrégé du Recensement de 2016, quatre mesures au niveau individuel ont été utilisées (i.e. basées sur les caractéristiques particulières liées au défunt, enregistrés dans le Recensement 2016) pour estimer les taux ventilés ainsi que les mesures d'inégalités. Ces mesures étaient les suivantes : mesure du faible revenu après impôt des ménages de Statistique Canada (26) (faible revenu ou non [groupe de référence]); type de logement (i.e. appartement dans un immeuble de moins de cinq étages, appartement dans un immeuble de cinq étages ou plus, appartement dans un duplex, maison en rangée, maison jumelée, versus maison individuelle non attenante [groupe de référence]) (6); type de ménage (i.e. personne vivant seule, couple avec enfants, couple sans enfant, ménage multigénérationnel, ménages sans famille de recensement composés de deux personnes ou plus, à l'exclusion des ménages multigénérationnels, " autre " ménage comptant une famille de recensement, versus ménage monoparental [groupe de référence]) (6) et taille du ménage (i.e. ménage de deux personnes, trois personnes, quatre personnes, cinq personnes ou plus versus ménage d'une seule personne [groupe de référence]) (6).

À partir de l'intégration des données provisoires de la Statistique de l'état civil et du FCCP+, trois mesures à l'échelle du quartier (27) ont été utilisées (i.e. des mesures des caractéristiques du quartier du défunt au moment du décès, s'appuyant sur les renseignements sur le code postal résidentiel) pour estimer les taux ventilés et les mesures d'inégalités. Ces mesures étaient les suivantes : résidence à I'intérieur ou à l'extérieur (groupe de référence) d'une région métropolitaine de recensement, soit un grand centre urbain de 100000 habitants ou plus (28); quintiles du revenu national après impôt par équivalent d'une personne seule (groupe de référence : quintile 5, revenus les plus élevés); et quintiles de la dimension de la composition ethnoculturelle nationale de l'Indice canadien de défavorisation multiple (groupe de référence: quintile 1, concentration la plus faible). Ce dernier est un indicateur composé qui saisit la concentration de personnes qui ont immigré récemment au Canada (au cours des cinq dernières années), qui appartiennent à une minorité visible, qui sont nées à l'extérieur du Canada ou qui n'ont aucune connaissance du français ou de l'anglais. Ce type de mesure peut aider à cerner les populations qui peuvent être particulièrement touchées par la discrimination et le désavantage systémiques. Par exemple, les personnes qui immigrent au Canada, particulièrement celles qui appartiennent à des minorités visibles, peuvent être victimes de formes structurelles ou institutionnelles de discrimination, notamment de discrimination raciale (i.e. de racisme "systémique » (29)), dans des domaines tels que le travail et le logement $(11,12)$.

Les données ont également été ventilées par sexe. Bien que seules les données sur le sexe (présumé à la naissance; "femme » ou " homme ») soient disponibles, cette étude se réfère ci-après aux inégalités selon le " sexe ou le genre ». Comme dans les rapports précédents (11), cette utilisation se fonde sur l'hypothèse que les inégalités de mortalité liée à la COVID-19 entre les hommes et les femmes, comme pour d'autres conditions de santé, sont déterminées par des facteurs liés à la fois au sexe biologique et au genre (11). 


\section{SURVEILLANCE}

\section{Analyses}

Les taux globaux et selon le sexe ou le genre ont été normalisés selon l'âge par la méthode directe, sur la base de la population canadienne standard de 2011, en utilisant des intervalles d'âge de cinq ans (30). Les détails sur les groupes d'âge, les formules et les poids ont été décrits précédemment (30). Les taux ont été normalisés en fonction de l'âge pour permettre la comparaison entre les groupes qui peuvent présenter des différences dans la structure d'âge $(30,31)$. Les intervalles de confiance pour ces taux ont été fixés à $95 \%$ et ont été calculés en utilisant l'erreur type du taux normalisé (les détails et les formules sont fournis dans le tableau supplémentaire $\mathbf{S 1}$ ). (32) Les taux normalisés selon l'âge et les estimations des intervalles de confiance ont été réalisés à l'aide des logiciels SAS 9.4 (33) et SAS Enterprise Guide 7.1 (34).

Pour évaluer les inégalités relatives et absolues en matière de mortalité liée à la COVID-19, les différences et les ratios de taux ont été estimés entre les sous-groupes, globalement et en fonction du sexe et du genre (selon les principes de l'analyse fondée sur le sexe et le genre Plus; SGBA+) en soustrayant et en divisant les taux entre les sous-groupes, avec des intervalles de confiance à $95 \%$ estimés en utilisant l'erreur type du taux pour chaque groupe comparé (les formules sont fournies dans le tableau supplémentaire S1) $(35,36)$. Les figures ont été créées à l'aide du logiciel R (version 4.0.2) (37). Puisque les estimations relatives aux inégalités s'appuyaient que sur des analyses bivariées, les « valeurs-e » (ou « e-values » en anglais) (38) ont été estimées pour évaluer la sensibilité potentielle des résultats aux variables de confusion non mesurées. Les « valeurs-e » représentent la taille minimale d'une association entre un facteur de confusion non mesuré et à la fois les mesures de stratification sociale et le risque de mortalité liée à la COVID-19, dans le but d'expliquer un ratio de risque observé. La « valeur-e » a été estimée comme suit : $R_{\text {observé }}+\sqrt{ }\left\{R R_{\text {observé }}\right.$ * $\left.\left(R R_{\text {observé }}-1\right)\right\}(38)$. Des valeurs-e plus élevées indiquent que des associations entre les facteurs de confusion potentiels non mesurés et les variables mesurées devraient être relativement fortes pour expliquer complètement les inégalités observées (38).

\section{Résultats}

\section{Répartition de la mortalité liée à la COVID-19 dans les sous-populations}

Au début de la pandémie, entre le $1^{\text {er }}$ janvier et le 4 juillet 2020, les taux de mortalité liée à la COVID-19 variaient selon les sous-groupes au niveau individuel (tableau 1). Les taux les plus bas et les plus élevés observés dans l'ensemble des sous-groupes mesurés concernaient les personnes vivant dans deux types de logements, respectivement : les taux allaient de neuf décès (pour les résidents de maisons individuelles non attenantes) à 23 et 26 décès (pour les résidents d'appartements) pour 100000 habitants. Les taux étaient plus élevés chez les hommes que chez les femmes.

Tableau 1 : Taux de mortalité normalisé selon l'âge pour 100000 habitants chez les résidents de logements privés, entre le $1^{\mathrm{er}}$ janvier et le 4 juillet 2020, selon les facteurs de stratification au niveau individuel du Recensement de 2016, pour l'ensemble du Canada et par sexe

\begin{tabular}{|c|c|c|c|c|c|c|}
\hline \multirow{3}{*}{ Facteurs de stratification } & \multicolumn{6}{|c|}{ Taux de mortalité par 100000 habitants normalisé selon l'âge } \\
\hline & \multicolumn{2}{|c|}{ Tous } & \multicolumn{2}{|c|}{ Femmes } & \multicolumn{2}{|c|}{ Hommes } \\
\hline & $\begin{array}{c}\text { Taux pour } \\
100000 \\
\text { habitants }\end{array}$ & IC $95 \%$ & $\begin{array}{c}\text { Taux pour } \\
100000 \\
\text { habitants }\end{array}$ & IC $95 \%$ & $\begin{array}{c}\text { Taux pour } \\
100000 \\
\text { habitants }\end{array}$ & IC $95 \%$ \\
\hline \multicolumn{7}{|l|}{ Statut de mesure de faible revenu (après impôt) } \\
\hline Personnes n'ayant pas un faible revenu & 14 & 13,14 & 11 & 10,11 & 18 & 17,19 \\
\hline Personnes à faible revenu & 19 & 18,20 & 15 & 14,17 & 27 & 25,30 \\
\hline \multicolumn{7}{|l|}{ Type de logement privé } \\
\hline Maison individuelle non attenante & 9 & 9,10 & 7 & 7,8 & 11 & 11,12 \\
\hline Maison en rangée & 13 & 11,15 & 9 & 7,11 & 19 & 15,22 \\
\hline Maison jumelée & 16 & 13,18 & 12 & 9,15 & 20 & 16,24 \\
\hline Appartement dans un immeuble de cinq étages ou plus & 23 & 21,24 & 18 & 16,19 & 33 & 30,35 \\
\hline Appartement dans un immeuble de moins de cinq étages & 24 & 23,26 & 18 & 16,20 & 36 & 33,39 \\
\hline Appartement dans un duplex & 26 & 23,29 & 19 & 16,21 & 37 & 32,42 \\
\hline \multicolumn{7}{|l|}{ Type de ménage } \\
\hline Famille monoparentale & 13 & 12,15 & 12 & 10,13 & 19 & 14,23 \\
\hline Ménage multigénérationnel & 14 & 13,16 & 13 & 11,15 & 17 & 14,20 \\
\hline Personne vivant seule & 15 & 14,15 & 11 & 11,12 & 22 & 21,24 \\
\hline Autre ménage comptant une famille de recensement ${ }^{\mathrm{a}}$ & 15 & 13,17 & 13 & 11,16 & 16 & 13,20 \\
\hline
\end{tabular}


Tableau 1 : Taux de mortalité normalisé selon l'âge pour 100000 habitants chez les résidents de logements privés, entre le $1^{\text {er }}$ janvier et le 4 juillet 2020, selon les facteurs de stratification au niveau individuel du Recensement de 2016, pour l'ensemble du Canada et par sexe (suite)

\begin{tabular}{|c|c|c|c|c|c|c|}
\hline \multirow{3}{*}{ Facteurs de stratification } & \multicolumn{6}{|c|}{ Taux de mortalité par 100000 habitants normalisé selon l'âge } \\
\hline & \multicolumn{2}{|c|}{ Tous } & \multicolumn{2}{|c|}{ Femmes } & \multicolumn{2}{|c|}{ Hommes } \\
\hline & $\begin{array}{c}\text { Taux pour } \\
100000 \\
\text { habitants }\end{array}$ & IC $95 \%$ & $\begin{array}{c}\text { Taux pour } \\
100000 \\
\text { habitants }\end{array}$ & IC $95 \%$ & $\begin{array}{c}\text { Taux pour } \\
100000 \\
\text { habitants }\end{array}$ & IC $95 \%$ \\
\hline \multicolumn{7}{|l|}{ Type de ménage (suite) } \\
\hline Couple sans enfant & 16 & 16,17 & 14 & 12,15 & 18 & 17,19 \\
\hline Couple avec enfants & 19 & 17,22 & 10 & 7,14 & 24 & 20,27 \\
\hline $\begin{array}{l}\text { Ménage sans famille de recensement de deux personnes ou plus } \\
\text { (sauf multigénérationnel) }\end{array}$ & 23 & 20,27 & 19 & 15,23 & 32 & 25,39 \\
\hline \multicolumn{7}{|l|}{ Taille du ménage } \\
\hline 1 personne & 15 & 14,15 & 11 & 11,12 & 22 & 21,24 \\
\hline 2 personnes & 15 & 15,16 & 12 & 11,13 & 18 & 17,19 \\
\hline 3 personnes & 15 & 14,17 & 11 & 9,12 & 21 & 18,24 \\
\hline 4 personnes & 14 & 11,16 & 11 & 9,14 & 16 & 12,19 \\
\hline 5 personnes ou plus & 17 & 15,19 & 15 & 12,17 & 20 & 16,23 \\
\hline
\end{tabular}

${ }^{a}$ Cela représente tous les ménages qui ne sont pas multigénérationnels où il y a une famille de recensement avec des personnes supplémentaires ou plus d'une famille de recensement

Entre le $1^{\text {er }}$ janvier et le 31 août 2020, les taux de mortalité liée à la COVID-19 variaient également selon les sous-groupes au niveau des quartiers (tableau 2). Pour 100000 habitants, les taux variaient de quatre décès (pour les résidents en dehors des grands centres urbains) à 33 à 37 décès (pour les résidents des grands centres urbains, des quartiers ayant les plus faibles revenus et ceux ayant la plus forte concentration de composition ethnoculturelle). Les taux dans ces populations étaient aussi plus élevés chez les hommes que chez les femmes.

Tableau 2 : Taux de mortalité normalisé selon l'âge pour 100000 habitants parmi l'ensemble des résidents, du $1^{\mathrm{er}}$ janvier au 31 août 2020, selon les facteurs de stratification au niveau des quartiers du Recensement de 2016, pour l'ensemble du Canada et par sexe

\begin{tabular}{|c|c|c|c|c|c|c|}
\hline \multirow{3}{*}{ Facteurs de stratification } & \multicolumn{6}{|c|}{ Taux de mortalité par 100000 habitants normalisé selon l'âge } \\
\hline & \multicolumn{2}{|c|}{ Tous } & \multicolumn{2}{|c|}{ Femmes } & \multicolumn{2}{|c|}{ Hommes } \\
\hline & $\begin{array}{c}\text { Taux pour } \\
100000 \\
\text { habitants }\end{array}$ & IC $95 \%$ & $\begin{array}{c}\text { Taux pour } \\
100000 \\
\text { habitants }\end{array}$ & IC $95 \%$ & $\begin{array}{c}\text { Taux pour } \\
100000 \\
\text { habitants }\end{array}$ & IC $95 \%$ \\
\hline \multicolumn{7}{|l|}{ Région métropolitaine de recensement (RMR) } \\
\hline $\begin{array}{l}\text { Dans les grands centres urbains (région métropolitaine de } \\
\text { recensement, RMR) }\end{array}$ & 33 & 32,34 & 29 & 28,29 & 39 & 38,41 \\
\hline En dehors des grands centres urbains (en dehors des RMR) & 4 & 3,4 & 3 & 2,3 & 5 & 4,5 \\
\hline \multicolumn{7}{|l|}{ Composition ethnoculturelle } \\
\hline Quintile 1 (concentration plus faible) & 16 & 15,17 & 14 & 13,15 & 18 & 17,20 \\
\hline Quintile 2 & 13 & 12,14 & 12 & 11,13 & 14 & 13,16 \\
\hline Quintile 3 & 19 & 18,20 & 16 & 15,17 & 22 & 20,24 \\
\hline Quintile 4 & 30 & 29,31 & 25 & 24,27 & 37 & 35,39 \\
\hline Quintile 5 & 37 & 35,38 & 31 & 30,33 & 44 & 42,47 \\
\hline \multicolumn{7}{|l|}{ Revenu des quartiers après impôt } \\
\hline Quintile 1 (revenus plus faibles) & 37 & 36,39 & 30 & 29,32 & 48 & 46,50 \\
\hline Quintile 2 & 20 & 19,20 & 16 & 15,17 & 24 & 22,25 \\
\hline Quintile 3 & 20 & 19,21 & 18 & 17,20 & 22 & 20,24 \\
\hline Quintile 4 & 18 & 17,19 & 16 & 15,17 & 21 & 20,23 \\
\hline Quintile 5 & 17 & 16,18 & 16 & 15,18 & 18 & 17,20 \\
\hline
\end{tabular}




\section{Inégalités absolues et relatives en matière de mortalité liée à la COVID-19 au sein des sous-groupes}

Entre le 1er janvier et le 4 juillet 2020, parmi les sous-groupes mesurés, les plus grandes inégalités absolues de mortalité liée à la COVID-19 parmi les résidents de logements privés ont été observées entre les résidents d'appartements (dans des duplex ou des immeubles à plusieurs étages) et les résidents de maisons individuelles non attenantes. II y avait 14 à 17 décès de plus pour 100000 (taux 2,5 à 2,8 fois plus élevés) chez les résidents d'appartements par rapport aux résidents de maisons individuelles non attenantes (figure 1) (les données présentées dans les figures 1 à 4 sont disponibles dans les tableaux supplémentaires S2 à S5, respectivement). Des inégalités moins importantes ont été observées entre les personnes vivant dans $d$ 'autres types d'habitation (maisons en rangée et maisons jumelées) et celles vivant dans des maisons individuelles non attenantes (le ratio des taux observés allait de 1,4 à 1,7, soit des différences de taux de 4 à 6 décès supplémentaires pour 100000 habitants). De même, des inégalités moins importantes ont été observées dans les sous-groupes pour type de ménage et statut de faible revenu; le ratio des taux observés allait de 1,1 à 1,8 , et les différences de taux d'un à dix décès supplémentaires pour 100000 dans ces sous-groupes (figure 1). II y avait peu ou pas de différences dans les taux selon la taille des ménages (comme l'indique le chevauchement des intervalles de confiance à $95 \%$ de la valeur nulle) (figure 1). Des analyses de sensibilité de la "valeur-e » ont été menées pour évaluer le risque de biais de confusion sur ces estimations bivariées d'inégalité. Les résultats suggèrent que les inégalités observées dans le risque de mortalité liée à la COVID-19 en fonction du statut de faible revenu, du type de ménage et du type d'habitation pourraient être entièrement expliquées par un facteur de confusion non mesuré qui aurait une association de $R R=2,1$ à 5,0 (selon les groupes sociaux), à la fois avec ces dernières mesures d'exposition et le risque de la mortalité liée à la COVID-19, respectivement (tableau supplémentaire S6). En d'autres termes, il faudrait que le facteur de confusion non mesuré ait une association plus forte que celles observées pour les facteurs mesurés dans cette étude (figure 1).

Figure 1 : Différences de taux et ratios des taux de mortalité normalisés selon l'âge (pour 100000 habitants) en fonction des caractéristiques individuelles, du $1^{\text {er }}$ janvier au 4 juillet 2020
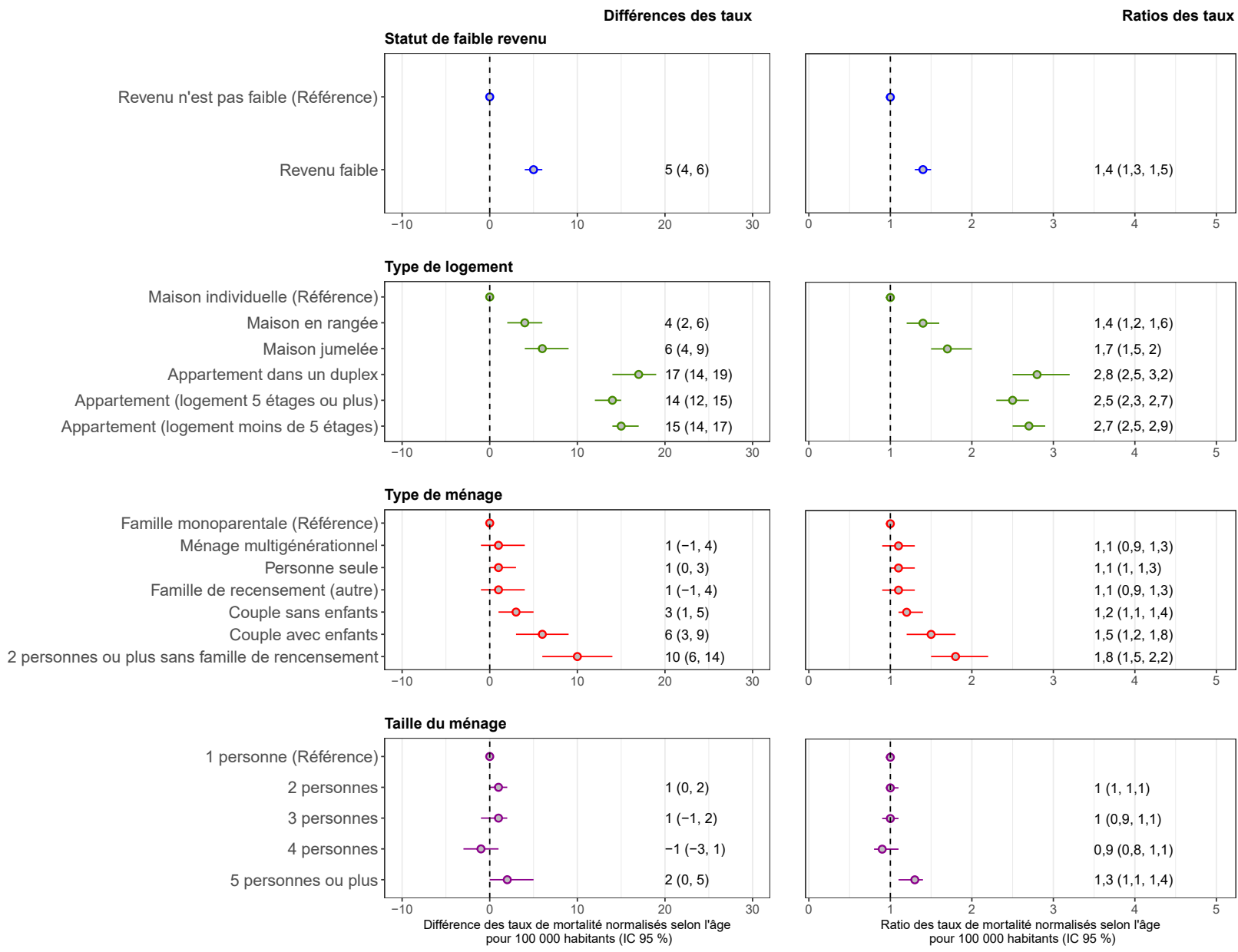
forte concentration de composition ethnoculturelle (quintiles 4 et 5 par rapport au quintile 1) et 20 décès de plus (taux 2,1 fois plus élevés) dans les quartiers ayant les plus faibles revenus (quintile 1 par rapport au quintile 5) (figure 2). Des analyses de sensibilité ont suggéré que ces dernières associations ne pouvaient être entièrement expliquées que par un facteur de confusion non mesuré, avec une association de $R R=3,2$ à 18,5 , selon les groupes sociaux, à la fois avec ces dernières expositions et le risque de mortalité lié à la COVID-19, respectivement (tableau supplémentaire $\mathrm{S7}$ ).

quartier. Pour 100000 habitants, il y avait 14 à 21 décès de plus (taux 1,9 à 2,3 fois plus élevés) dans les quartiers ayant la plus

Figure 2 : Différences de taux et ratios des taux de mortalité normalisés selon l'âge (pour 100000 habitants) en fonction des caractéristiques des quartiers, du 1 er janvier au 31 août 2020
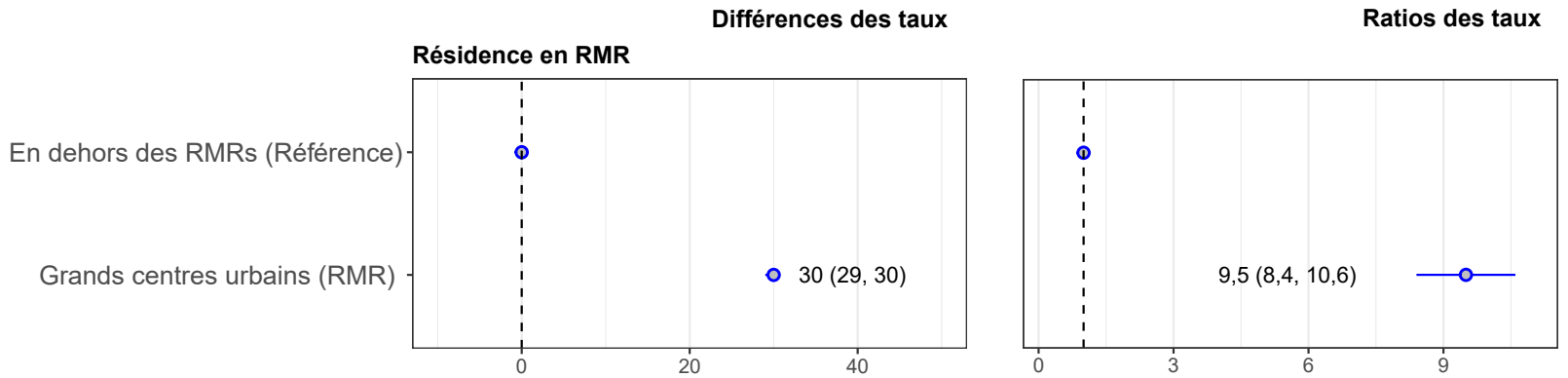

Composition ethnoculturelle
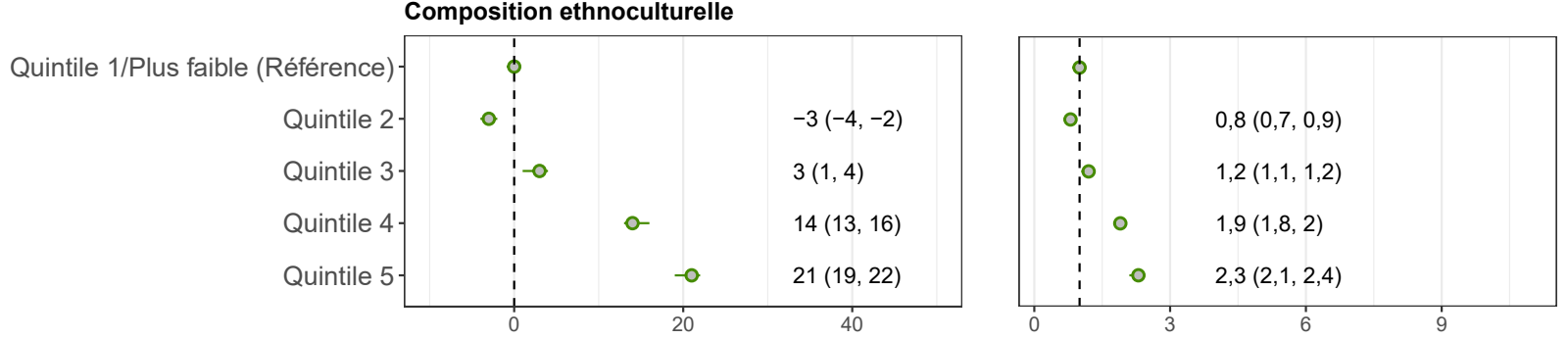

Revenu du quartier
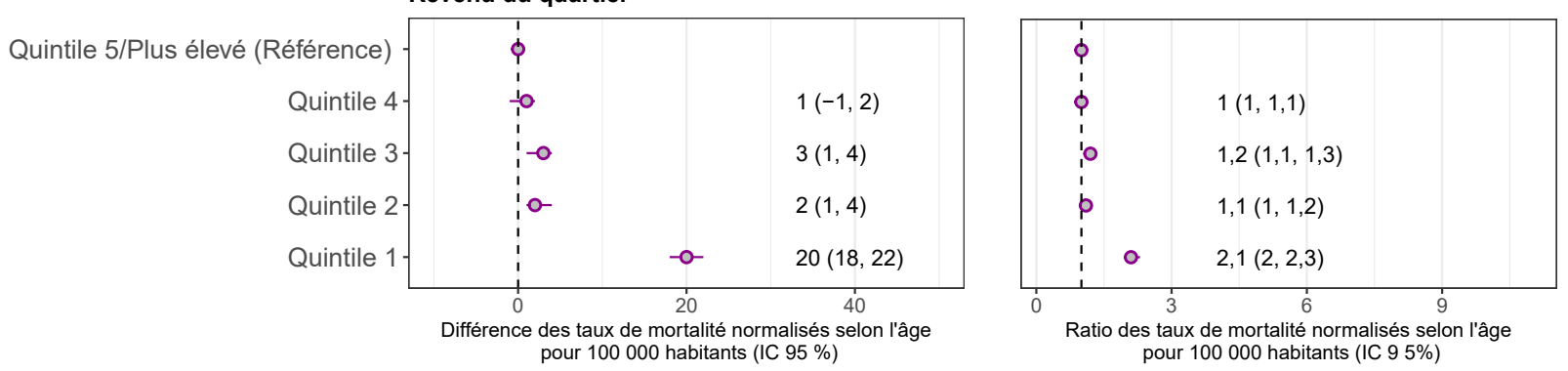

Abréviation : RMR, région métropolitaine de recensement 
Les différences de taux pour les autres groupes de quintiles de revenu du quartier (quintiles 2 à 4) et les groupes de quintiles de composition ethnoculturelle (quintiles 2 à 3 ) variaient d'un à trois décès pour 100000 habitants (ratios de 0,8 à 1,2), et plusieurs des intervalles de confiance à $95 \%$ chevauchaient la valeur nulle (figure 2).

\section{Inégalités entre les hommes et les femmes en matière de mortalité liée à la COVID-19 au sein des sous-populations}

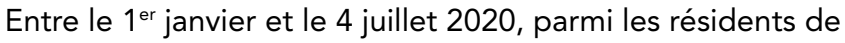
logements privés, les plus grandes inégalités de mortalité entre les hommes et les femmes concernaient les habitants d'appartements (différence de 15 à 18 décès supplémentaires pour 100000 habitants, ratios hommes-femmes de 1,8 à 2) (figure 3). Dans les autres sous-groupes de type d'habitation, les différences de taux allaient de quatre à dix décès pour 100000 habitants (ratio hommes-femmes de 1,6 à 2,1) (figure 3).

Figure 3 : Différences et ratios de taux de mortalité normalisés selon l'âge entre les hommes et les femmes (groupe de référence) par sous-groupes au niveau individuel, du $1^{\text {er }}$ janvier au 4 juillet 2020

Différences des taux, hommes vs, femmes

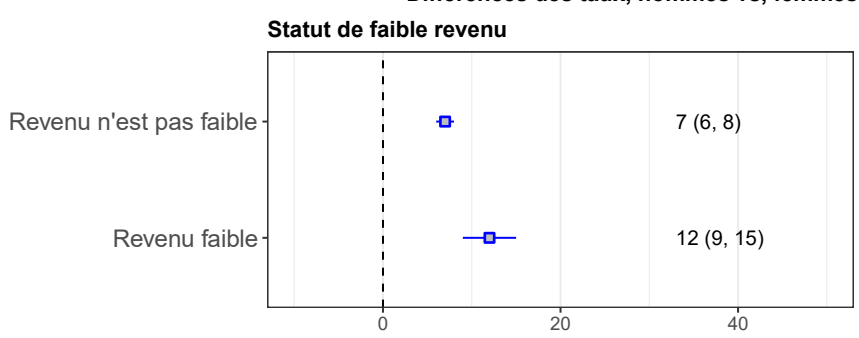

Type de logement
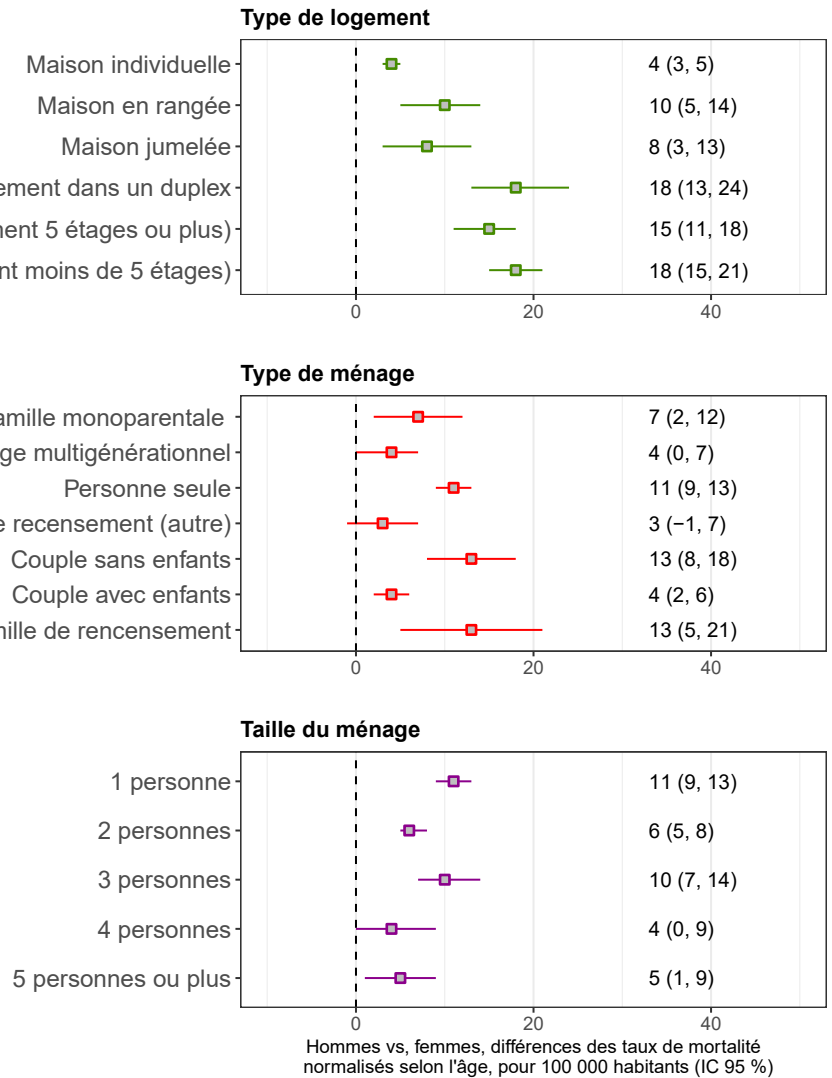

Ratio des taux, hommes vs, femmes
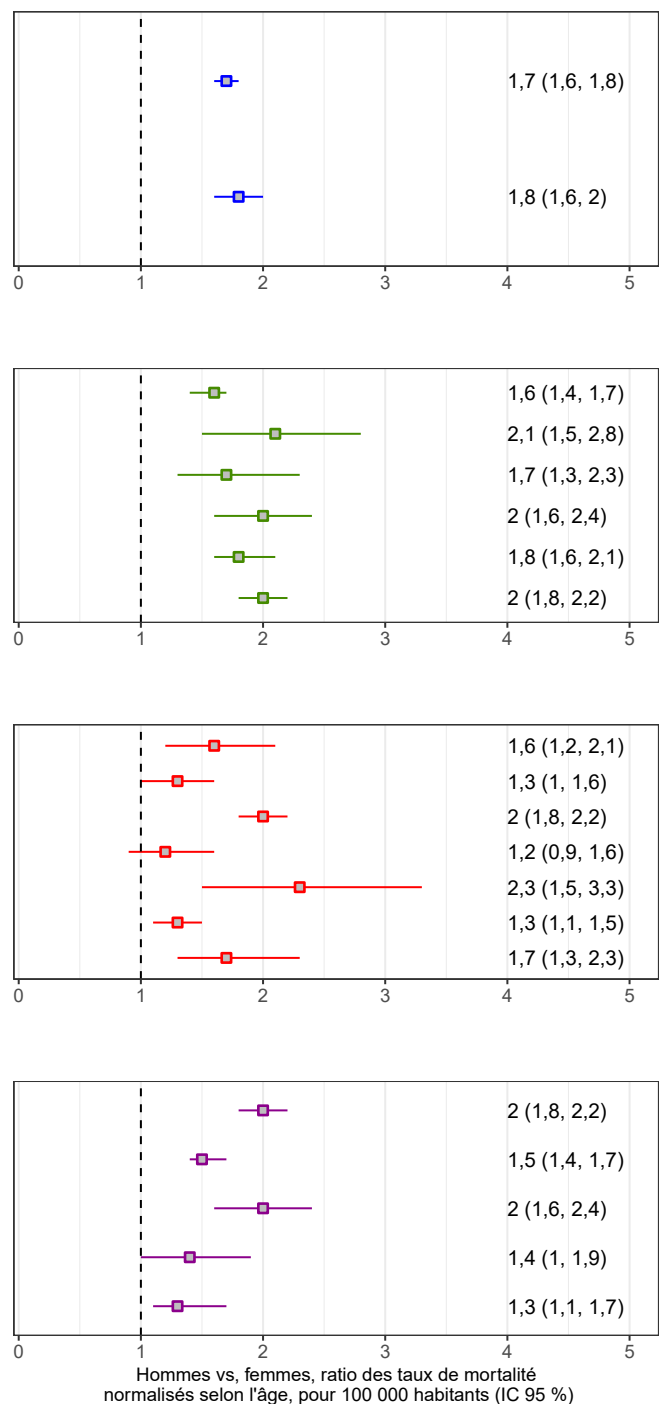
Parmi les types de ménages, les plus grandes inégalités entre les sexes étaient observées au sein des ménages d'une personne, des ménages sans famille de recensement de deux personnes ou plus et des couples avec enfants (différences de taux de 11 à 13 pour 100000 habitants, ratio hommes-femmes de 1,7 à 2,3) (figure 3). Dans les autres types de ménages, les différences allaient de trois à sept pour 100000 habitants (ratio hommesfemmes de 1,2 à 1,6), avec plusieurs intervalles de confiance à $95 \%$ chevauchant la valeur nulle (figure 3 ). Les hommes ont connu 12 décès de plus pour 100000 habitants que les femmes (ratio hommes-femmes de 1,8) dans les groupes à faible revenu, contre sept décès de plus pour 100000 habitants (ratio hommesfemmes de 1,7) dans les groupes qui ne sont pas à faible revenu (figure 3). Enfin, par rapport aux femmes, les hommes ont connu entre 6 et 11 décès de plus dans les ménages d'une à trois personnes (taux 1,5 à 2 fois plus élevés) (figure 3 ). Dans les autres sous-groupes liés à la taille de ménage, les intervalles de confiance à $95 \%$ pour les différences de taux et les ratios étaient près de la valeur nulle (figure 3 ).
De même, entre le $1^{\mathrm{er}}$ janvier et le 31 août 2020, les inégalités selon le sexe et le genre variaient en fonction des ventilations au niveau des quartiers. Il y avait 11 décès de plus chez les hommes que chez les femmes pour 100000 habitants dans les régions métropolitaines de recensement, comparativement à deux décès de plus chez les hommes pour 100000 habitants à l'extérieur des centres urbains (figure 4). La différence de taux de mortalité entre les hommes et les femmes était la plus importante dans les quartier ayant les plus faibles revenus ou ayant la plus forte concentration de composition ethnoculturelle : pour 100000 habitants, il y avait 18 décès de plus chez les hommes dans le quintile 1 de revenu (taux 1,6 fois plus élevé que chez les femmes) et 13 décès de plus chez les hommes dans le quintile 5 de composition ethnoculturelle (taux 1,4 fois plus élevé que chez les hommes que chez les femmes) (figure 4).

Figure 4 : Différences et ratios de taux de mortalité normalisée selon l'âge entre les hommes et les femmes (groupe de référence) par sous-groupes au niveau des quartiers, du $1^{\text {er }}$ janvier au 31 août 2020
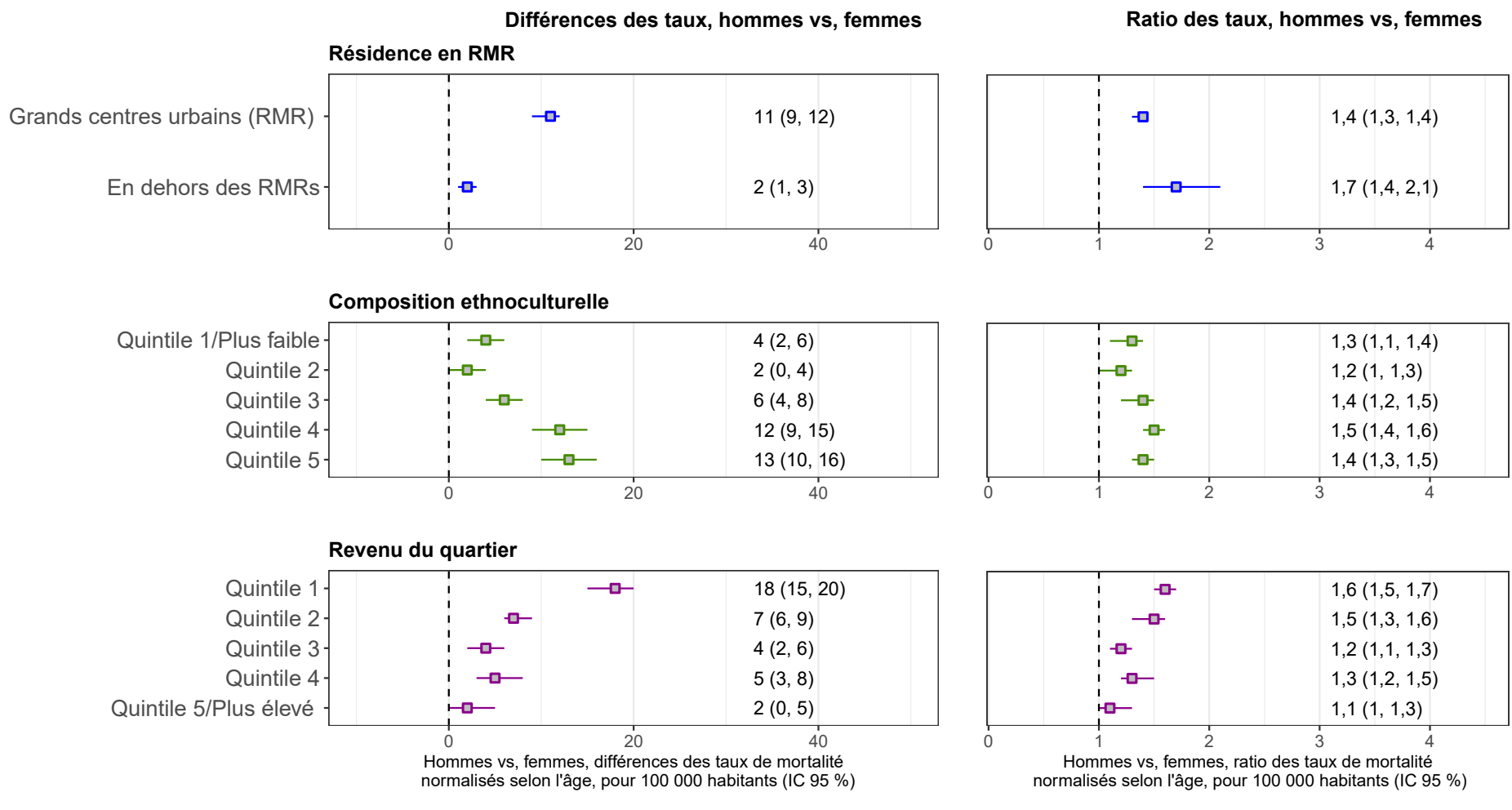

Abréviation : RMR, région métropolitaine de recensement 


\section{Discussion}

Cette étude visait à donner un aperçu des inégalités aux niveaux individuel et de quartier en matière de mortalité liée à la COVID-19 au Canada, au début de la pandémie. Au niveau individuel, les plus grandes inégalités de mortalité ont été observées entre les résidents d'appartements et ceux de maisons individuelles non attenantes. Au niveau des quartiers, de grandes inégalités ont été observées entre les personnes vivant dans les grands centres urbains, dans les quartiers ayant les plus faibles revenus et ayant la plus forte concentration de composition ethnoculturelle, par rapport aux groupes de référence respectifs. Les inégalités entre les taux de mortalité des hommes et des femmes étaient également plus élevées dans chacun des sousgroupes ci-dessus. Ces résultats reflètent le fardeau global de mortalité attribuable à la COVID-19 plus élevé que subissent les populations défavorisées.

Les inégalités observées, notamment en ce qui concerne les revenus et la composition ethnoculturelle, sont conformes aux résultats précédemment obtenus au Canada aux niveaux régional (1-3), provincial $(4,5)$ et national $(6,7,39)$. De plus, les inégalités entre les hommes et les femmes et le revenu à l'échelle des quartiers concordent également à ce qui a été observé pour $d$ 'autres maladies infectieuses et chroniques ainsi que pour la mortalité globale au Canada $(11,40,41)$.

Des rapports antérieurs ont souligné que les inégalités en matière de mortalité attribuable à la COVID-19 peuvent s'expliquer par des différences sociales et économiques dans I'infection par le SRAS-CoV-2 (13-15), et à la répartition des facteurs de risque de mortalité, notamment la prévalence des maladies chroniques ainsi que l'accès et l'utilisation des services de santé (9). Par exemple, les inégalités systémiques dans les conditions de vie et de travail peuvent entraîner une répartition inéquitable des infections et des risques de morbidité (8). Les inégalités plus importantes entre les hommes et les femmes en matière de mortalité liée à la COVID-19 observées dans certains sous-groupes sont probablement un indicateur de l'interaction entre les facteurs immunologiques liés au sexe (42) et les expériences domestiques et professionnelles liées au genre qui façonnent le risque d'infection et de morbidité, y compris les comportements à risque (e.g. le tabagisme, le faible recours aux services de soins de santé (11) et la prévalence de maladies chroniques (42)).

De plus, les déterminants sociaux potentiels des issues de santé découlant de la COVID-19 peuvent inclure les mesures de santé publique, qui peuvent avoir des effets différents sur les populations, notamment en ce qui concerne la transmission du SRAS-CoV-2. Par exemple, un rapport de la Toronto Foundation indiquait comment la fermeture de lieux de travail non essentiels était associée à des taux de transmission du SRAS-CoV-2 plus faibles dans les quartiers ayant des revenus plus élevés, où davantage de résidents pouvaient travailler à domicile (43).
Cette politique semble être moins efficace dans les quartiers ayant de plus faibles revenus et une plus forte concentration de populations de minorités visibles (43). Il est courant que les interventions de la santé publique d'envergure universelle aient des effets variables si certains groupes socioéconomiques sont confrontés à des obstacles structurels qui les empêchent de profiter des avantages de ces interventions $(44,45)$, comme l'incapacité de travailler à domicile, (46) l'absence de temps libre pour accéder à des services ou ressources, ou les barrières linguistiques (11). Comme tel, certains chercheurs ont notamment stipulé que les stratégies qui proposent une combinaison d'approches universelle et ciblées, fondées sur les besoins proportionnels des populations, permettraient de surmonter ces limites (47).

Par ailleurs, le fait que le fardeau de la mortalité attribuable à la COVID-19 diffère dans certains groupes, mais qu'il ne diffère pas dans d'autres groupes, suppose que ces inégalités de mortalité peuvent être évitées. Par conséquent, ces inégalités peuvent être considérées comme inéquitables (48). À la lumière de ces résultats, il est évident que des efforts doivent être déployés pour avancer l'équité en santé au Canada pendant cette pandémie et à l'avenir dans le but de prévenir ces inégalités, conformément à ce qui est proposé dans le rapport intitulé Les principales inégalités en matière de santé au Canada (11).

\section{Limites}

Cette étude présente plusieurs limites. Premièrement, cette analyse visait à mieux comprendre les différences de mortalité entre les sous-populations, en utilisant les meilleures sources de données disponibles. Cependant, tel qu'indiqué précédemment, les données provisoires utilisées dans ce rapport ont probablement sous-estimé les taux de mortalité liée à la COVID-19. Les taux rapportés ne tiennent pas compte de tous les décès liés à la COVID-19 survenus au Canada pendant la période d'étude. À ce stade, il est impossible de mesurer si et comment la sous-déclaration de décès aurait pu influencer l'ampleur des inégalités observées. De plus, il n'était pas possible d'estimer, avec les données disponibles, comment les différences de sous-déclaration entre les groupes, ou les changements spatio-temporels de la sous-déclaration ou des taux de transmission, auraient pu influencer l'ampleur des inégalités au fil du temps. Deuxièmement, en raison des limitations de l'accès aux données, cette étude n'a pas exploré les interactions entre les mesures; également, aucune analyse multivariée n'a été réalisée pour identifier les mécanismes spécifiques par lesquelles ces inégalités se manifestent. Bien que les analyses de sensibilité effectuées suggèrent une vulnérabilité minimale à modérée à l'égard des biais de confusion potentiels, des analyses multivariée ultérieures sont nécessaires afin de combler ces lacunes. Troisièmement, les caractéristiques personnelles ou à l'échelle des quartiers peuvent avoir changé entre le moment de la collecte du Recensement de 2016 et celui où les décès sont survenus. Au moment de réaliser cette étude, il n'était pas possible d'estimer comment ce facteur 
aurait pu influencer les estimations des inégalités. Également, cette étude n'a pas permis de distinguer les décès (parmi les bases de données des décès intégrées avec les données à l'échelle des quartiers ainsi que les inégalités qui en découlent) étant survenus auprès de résidents d'établissements de soins de longue durée de ceux survenus dans les logements privés. Ainsi, il est important de considérer ces aspects dans le cadre d'études futures. Enfin, cette étude n'a pas exploré plusieurs autres déterminants sociaux, notamment le genre, l'identité Autochtone, ou la race/l'ethnicité, puisque ces données n'étaient pas disponibles. L'exploration de ces déterminants sociaux, et des inégalités par province et territoire, à des moments ultérieurs de la pandémie, notamment après l'apparition des variants préoccupants du virus (49) et des campagnes de vaccination, $s$ 'avère un autre domaine important de recherches futures.

\section{Conclusion}

Le fardeau de mortalité liée à la COVID-19 entre janvier et juillet/août 2020 n'a pas été vécu de la même manière pour toutes les populations et communautés du Canada. Cette étude souligne l'influence des déterminants sociaux de la santé et des inégalités socioéconomiques dans la répartition inéquitable du fardeau de la COVID-19, et la nécessité de prendre en considération ces facteurs dans les analyses futures, afin de préparer une réponse à la pandémie axée sur l'équité en santé.

\section{Déclaration des auteurs}

A. B. - Conceptualisation de l'étude, réalisation des analyses des inégalités absolues et relatives, interprétation des données, rédaction et révision du manuscrit

S. Y. P. - Conceptualisation de l'étude, réalisation des analyses des inégalités absolues et relatives, rédaction et commentaires sur le manuscrit

N. A. - Estimation des taux ventilés et fourni des commentaires sur le manuscrit

F. J. Y. - Estimation des taux ventilés et fourni des commentaires sur le manuscrit

R. S. - Estimation des taux ventilés et fourni des commentaires sur le manuscrit

C. S. - Conceptualisation de l'étude et fourni des commentaires sur le manuscrit

\section{Intérêts concurrents}

Aucun.

\section{Remerciements}

Cette analyse est le fruit de l'Initiative pancanadienne sur les inégalités en santé. Créée en 2012 et dirigée par l'Agence de la santé publique du Canada (l'Agence), I'Initiative pancanadienne sur les inégalités en santé implique une collaboration entre l'Agence, Statistique Canada, le Réseau pancanadien de santé publique, I'Institut canadien d'information sur la santé et le Centre de gouvernance de l'information des Premières Nations. Les rapports antérieurs de l'Initiative pancanadienne sur les inégalités en santé incluaient un outil interactif en ligne de données sur les inégalités en santé (outil de données sur les inégalités en santé) et Les principales inégalités en santé au Canada de 2018 : A National Portrait. Nous tenons à remercier Scott Van Millingen et Hongbo Liang pour leur contribution au développement de l'outil de données liées à la COVID-19 de I'Initiative pancanadienne sur les inégalités en santé et de l'outil de données sur les inégalités de santé.

\section{Financement}

Ce travail a été soutenu par l'Agence de santé publique du Canada.

\section{Matériel supplémentaire}

Ces documents sont accessibles dans le dossier

Tableaux supplémentaires

Tableau supplémentaire S1

Tableau supplémentaire S2

Tableau supplémentaire S3

Tableau supplémentaire S4

Tableau supplémentaire S5

Tableau supplémentaire S6

Tableau supplémentaire S7

\section{Références}

1. Toronto Public Health. COVID-19: Pandemic Data. Toronto (ON): TPH; June 2021 (accédé 2021-10-27). https://www. toronto.ca/home/covid-19/covid-19-pandemic-data/

2. Ottawa Public Health. COVID-19 and Racial Identity in Ottawa; February to August 2020. Toronto (ON): TPH; November 2020 (accédé 2021-10-27). https://www. ottawapublichealth.ca/en/reports-research-and-statistics/ resources/Documents/covid-19/Special-Focus/Report--COVID-19-and-Racial-Identity-in-Ottawa-2020.pdf

3. Direction régionale de la santé publique de Montréal. Le point sur la santé des Montréalais en période de pandémie. Montréal (OC) : Santé Montréal; 2020 (accédé 2021-10-27). https://santemontreal.qc.ca/population/coronaviruscovid-19/situation-du-coronavirus-covid-19-a-montreal/pointsante/populations-racisees/

4. ICES. ICES releases report on COVID-19 in immigrants, refugees and other newcomers in Ontario. ICES; September 2020 (accédé 2021-10-27). https://www.ices.on.ca/ Newsroom/Announcements-and-Events/2020/ICES-releasesreport-on-COVID-19-in-Immigrants-Refugees-and-OtherNewcomers-in-Ontario 
5. Chung $H$, Fung $K$, Ferreira-Legere LE, Chen B, Ishiguro L, Kalappa G, Gozdyra P, Campbell T, Paterson JM, Bronskill SE, Kwong JC, Guttmann A, Azimaee M, Vermeulen MJ, Schull MJ. COVID-19 Laboratory Testing in Ontario: Patterns of Testing and Characteristics of Individuals Tested, as of April 30; 2020. ICES, 2020 (accédé 2021-10-27). https://www.ices.on.ca/Publications/Atlasesand-Reports/2020/COVID-19-Laboratory-Testing-in-Ontario

6. Statistique Canada. Yang F, Aitken N. Les personnes qui vivaient en appartement ou au sein d'un ménage plus nombreux étaient plus à risque de mourir de la COVID-19 au cours de la première vague de la pandémie. Ottawa (ON) : StatCan; 2021 (accédé 2021-10-27). https://www150.statcan. gc.ca/n1/pub/45-28-0001/2021001/article/00004-fra.htm

7. Statistique Canada. StatCan et la COVID-19 : Des données aux connaissances, pour bâtir un Canada meilleur. Subedi R, Greenberg L, Turcotte M. Taux de mortalité attribuable à la COVID-19 dans les quartiers ethnoculturels du Canada. Ottawa (ON) : StatCan; 2020 (accédé 2021-10-27).

https://www150.statcan.gc.ca/n1/pub/45-28-0001/2020001/ article/00079-fra.htm

8. World Health Organization. Solar O, Irwin A. A conceptual framework for action on the social determinants of health. Geneva, CH; WHO; 2010 (accédé 2021-10-27). https://www. who.int/sdhconference/resources/Conceptualframeworkforac tiononSDH_eng.pdf

9. Agence de la santé publique du Canada. Du risque à la résilience : Une approche axée sur l'équité concernant la COVID-19. Rapport de l'administratrice en chef de la santé publique. Ottawa (ON) : ASPC; 2020 (accédé 2021-10-27). https://www.canada.ca/fr/sante-publique/organisation/ publications/rapports-etat-sante-publique-canadaadministrateur-chef-sante-publique/du-risque-resilienceapproche-equite-covid-19.html2

10. Mishra S, Kwong JC, Chan AK, Baral SD. Understanding heterogeneity to inform the public health response to COVID-19 in Canada. CMAJ 2020;192(25):E684-5. DOI PubMed

11. Agence de la santé publique du Canada. Les principales inégalités en santé au Canada - Sommaire exécutif. Ottawa (ON) : ASPC; 2018 (accédé 2021-10-27). https://www. canada.ca/fr/sante-publique/services/publications/sciencerecherche-et-donnees/rapport-principales-inegalites-santecanada-sommaire-executif.htm

12. Walk RA, Bourne LS. Ghettos in Canada's cities? Racial segregation, ethnic enclaves and poverty concentration in Canadian urban areas. Can Geogr 2006;50(3):273-97. DOI

13. Bi Q, Wu Y, Mei S, Ye C, Zou X, Zhang Z, Liu X, Wei L, Truelove SA, Zhang T, Gao W, Cheng C, Tang X, Wu X, Wu Y, Sun B, Huang S, Sun Y, Zhang J, Ma T, Lessler J, Feng T. Epidemiology and transmission of COVID-19 in 391 cases and 1286 of their close contacts in Shenzhen, China: a retrospective cohort study. Lancet Infect Dis 2020;20(8):911-9. DOl PubMed
14. International Long Term Care Policy Network. Hsu AT, Lane N, Sinha SK, Dunning J, Dhuper M, Kahiel Z, Sveistrup H. Impact of COVID-19 on residents of Canada's long-term care homes-ongoing challenges and policy responses. ILTCPN; 2020 (accédé 2021-10-27).

https://ltccovid.org/wp-content/uploads/2020/05/LTCcovidcountry-reports_Canada_Hsu-et-al_May-10-2020-2.pdf

15. Leso V, Fontana L, lavicoli I. Susceptibility to Coronavirus (COVID-19) in Occupational Settings: The Complex Interplay between Individual and Workplace Factors. Int J Environ Res Public Health 2021;18(3):10. DOI PubMed

16. Statistique Canada. StatCan et la COVID-19 : Des données aux connaissances, pour bâtir un Canada meilleur. O'Brien $\mathrm{K}$, St-Jean M, Wood P, Willbond S, Phillips O, Currie D, Turcotte M. Comorbidités liées aux décès impliquant la COVID-19 au Canada. Ottawa (ON) : StatCan; 2020 (accédé 2021-10-27). https://www150.statcan.gc.ca/n1/pub/45-28-0001/2020001/ article/00087-fra.htm

17. Bryant T, Leaver C, Dunn J. Unmet healthcare need, gender, and health inequalities in Canada. Health Policy 2009;91(1):24-32. DOl PubMed

18. Blair A, Warsame K, Naik H, Byrne W, Parnia A, Siddiqi A. Identifying gaps in COVID-19 health equity data reporting in Canada using a scorecard approach. Can J Public Health 2021;112(3):352-62. DOI PubMed

19. Center for Research-Action on Race Relations. CRARR Calls on Federal and Quebec Governments to Collect COVID-19 Data Based on Race, Language, Income Level. Montreal (QC): CRARR; 2020 (accédé 2021-10-27). http://www.crarr. $\mathrm{org} / \mathrm{q}=$ node/20102

20. Béland F. Pandémie, iniquités, santé publique, information, interventions : I'échec canadien? Can J Public Health 2021;112(3):349-51. DOl PubMed

21. McKenzie K. Socio-demographic data collection and equity in covid-19 in Toronto. EClinicalMedicine 2021;34(100812):100812. DOl PubMed

22. Statistique Canada. Nombre provisoire de décès et surmortalité, janvier à août 2020. Ottawa (ON) : StatCan; 2020 (accédé 2021-11-03). https://www150.statcan.gc.ca/n1/ daily-quotidien/201028/dq201028b-fra.htm

23. Statistique Canada. Environnement de couplage de données sociales : Aperçu. Ottawa (ON) : StatCan; 2017 (accédé 2021-11-03). https://www.statcan.gc.ca/fr/ecds/apercu

24. Statistique Canada. Tableaux de données, Recensement de 2016. Population, logements et ménages (8) du Canada, provinces et territoires, recensements de 1981 à 2016. Numéro cataloque. 98-400-X2016013. Ottawa (ON): StatCan; 2016 (accédé 2021-11-03). https://www12.statcan. gc.ca/census-recensement/2016/dp-pd/dt-td/Rp-fra.cfm?TA $\mathrm{BID}=1 \& \mathrm{LANG}=\mathrm{F} \& A=\mathrm{R} \& A P A T H=3 \& D E T A I L=0 \& D I M=0 \& F L=$ $A \& F R E E=0 \& G C=01 \& G L=-1 \& G I D=1234492 \& G K=1 \& G R P=1$ $\& O=D \& P I D=109532 \& P R I D=10 \& P T Y P E=109445 \& S=0 \& S H O$ WALL $=0 \& S U B=0 \&$ Temporal $=2016 \&$ THEME $=116 \& \mathrm{VID}=0 \&$ $\mathrm{VNAMEE}=\& \mathrm{VNAMEF}=\& \mathrm{D} 1=0 \& \mathrm{D} 2=0 \& \mathrm{D} 3=0 \& \mathrm{D} 4=0 \& \mathrm{D} 5=0$ $\& D 6=$ 
25. Statistique Canada. Fichier de conversion des codes postaux MO plus (FCCP+). Ottawa (ON) : StatCan; 2021 (accédé 2021-10-27). https://www150.statcan.gc.ca/n1/fr/ catalogue/82F0086X

26. Statistique Canada. Série de documents de recherche Revenu. Les lignes de faible revenu : leur signification et leur calcul. Ottawa (ON) : StatCan; 2016 (accédé 2021-10-27). https://www150.statcan.gc.ca/n1/ pub/75f0002m/75f0002m2016002-fra.htm

27. Statistique Canada. Dictionnaire, Recensement de la population, 2016 : Aire de diffusion (AD). Ottawa (ON) : StatCan; 2016 (accédé 2021-05-17). https://www12.statcan. gc.ca/census-recensement/2016/ref/dict/geo021-fra.cfm

28. Statistique Canada. RMR et AR : définition détaillée. Ottawa (ON) : StatCan; 2018 (accédé 2021-05-17). https://www150. statcan.gc.ca/n1/pub/92-195-x/2011001/geo/cma-rmr/deffra.htm

29. Centre de collaboration nationale des déterminants de la santé. Le racisme et l'équité en santé : Parlons-en. Antigonish (N-E) : CCNDS; 2020 (accédé 2021-10-27). https://nccdh.ca/fr/resources/entry/lets-talk-racism-andhealth-equity

30. Statistique Canada. Statistiques de l'état civil, Base de données sur les décès - Glossaire. Ottawa (ON) : StatCan; 2017 (accédé 2021-11-03). https://www.statcan.gc.ca/fr/ programmes-statistiques/document/3233_D4_T9_V1

31. Statistique Canada. À la base des données ; Taux normalisés selon l'âge. Ottawa (ON) : StatCan; 2017 (accédé 2021-10-27). https://www.statcan.gc.ca/fr/quo/bdd/tnsa

32. Statistique Canada. Indicateurs de la santé : Qualité des données, concepts et méthodologie : Indicateurs de l'état de santé fondés sur les statistiques de l'état civil. Ottawa (ON): StatCan; 2016 (accédé 2021-11-03). https://www150. statcan.gc.ca/n1/pub/82-221-x/2013001/quality-qualite/ qual-fra.htm

33. SAS Institute. Base SAS 9.4 Procedures Guide (5 $5^{\text {th }}$ edition). SAS Institute Inc., USA; 2015. https://support.sas.com/ content/dam/SAS/support/en/documentation/third-partyreference/493971_9.4-indbag-5th-ed.pdf

34. SAS Institute. Statistics using SAS Enterprise Guide. Cambridge University Press, UK; 2009. DOI

35. Harvard School of Public Health. The Public Health Disparities Geocoding Project. The Public Health Disparities Geocoding Project Monograph: Analytic Methods. Boston (MA): HSPH; 2021 (accédé 2021-06-15). https://www.hsph. harvard.edu/thegeocodingproject/analytic-methods/

36. Rothman K, Greenland S. Modern Epidemiology. $2^{\text {nd }}$ ed. Philadelphia (PA): Lippincott William and Wilkins; 1998. https://www.rti.org/publication/modern-epidemiology-2ndedition
37. The R Project for Statistical Computing. R version 4.0.2; 2020. https://www.r-project.org/

38. VanderWeele TJ, Ding P. Sensitivity Analysis in Observational Research: introducing the E-Value. Ann Intern Med 2017;167(4):268-74. DOl PubMed

39. Statistics Canada. StatCan et la COVID-19 : Des données aux connaissances, pour bâtir un Canada meilleur. $\mathrm{Ng} \mathrm{E}$. Décès liés à la COVID-19 chez les immigrants : données probantes des premiers mois de la pandémie. Ottawa (ON) : StatCan; 2021 (accédé 2021-06-11). https://www150.statcan. gc.ca/n1/pub/45-28-0001/2021001/article/00017-fra.htm

40. Mustard CA, Etches J. Gender differences in socioeconomic inequality in mortality. J Epidemiol Community Health 2003;57(12):974-80. DOl PubMed

41. Rosella LC, Calzavara A, Frank JW, Fitzpatrick T, Donnelly PD, Henry D. Narrowing mortality gap between men and women over two decades: a registry-based study in Ontario, Canada. BMJ Open 2016;6(11):e012564. DOI PubMed

42. Maclntyre S, Hunt K. Socio-economic position, gender and health: how do they interact? J Health Psychol 1997;2(3):315-34. DOl PubMed

43. Toronto Foundation. Toronto Fallout Report: Half a year in the life of COVID-19. Toronto (ON): Toronto Foundation; 2020 (accédé 2021-11-03). https://torontofoundation.ca/wpcontent/uploads/2020/11/Toronto-Fallout-Report-2020.pdf

44. Rose G. Sick individuals and sick populations. Int J Epidemiol 2001;30(3):427-32. DOI PubMed

45. Rose G, Khaw KT, Marmot M. Rose's Strategy of Preventive Medicine. USA: Oxford University Press; 2008. DOI

46. Statistique Canada. Tableau 1 : Changement du principal lieu de travail selon le niveau de scolarité, Canada, 22 au 28 mars 2020. Ottawa (ON) : StatCan; 2020 (accédé 2021-06-11). https://www150.statcan.gc.ca/n1/dailyquotidien/200417/t001a-fra.htm

47. Marmot M, Allen J, Bell R, Bloomer E, Goldblatt P; Consortium for the European Review of Social Determinants of Health and the Health Divide. WHO European review of social determinants of health and the health divide. Lancet 2012;380(9846):1011-29. DOI PubMed

48. Whitehead $M$. The concepts and principles of equity and health. Health Promot Int 1991;6(3):217-28. DOI PubMed

49. Chagla Z, Ma H, Sander B, Baral SD, Mishra S. Characterizing the disproportionate burden of SARS-CoV-2 variants of concern among essential workers in the Greater Toronto Area, Canada. medRxiv 2021; 2021.03.22.21254127v2. https://www.medrxiv.org/content/10.1101/2021.03.22.2125 4127v2 